\section{D) Check for updates}

Cite this: Analyst, 2021, 146, 3197

\title{
Characterization of a liquid-core waveguide cell for studying the chemistry of light-induced degradation $\uparrow$
}

\author{
Iris Groeneveld, (D *a Suzan E. Schoemaker, ${ }^{a}$ Govert W. Somsen, ${ }^{a}$ Freek Ariese (D) ${ }^{b}$ \\ and Maarten R. van Bommel ${ }^{\mathrm{c}, \mathrm{d}}$
}

\begin{abstract}
Many organic compounds undergo changes under the influence of light. This might be beneficial in, for example, water purification, but undesirable when cultural-heritage objects fade or when food ingredients (e.g., vitamins) degrade. It is often challenging to establish a strong link between photodegradation products and their parent molecules due to the complexity of the sample. To allow effective study of lightinduced degradation (LID), a low-volume exposure cell was created in which solutes are efficiently illuminated (especially at low concentrations) while simultaneously analysed by absorbance spectroscopy. The new LID cell encompasses a gas-permeable liquid-core waveguide (LCW) connected to a spectrograph allowing collection of spectral data in real-time. The aim of the current study was to evaluate the overall performance of the LID cell by assessing its transmission characteristics, the absolute photon flux achieved in the LCW, and its capacity to study solute degradation in presence of oxygen. The potential of the LID set-up for light-exposure studies was successfully demonstrated by monitoring the degradation of the dyes eosin $\mathrm{Y}$ and crystal violet.
\end{abstract}

Received 12th February 2021 Accepted 30th March 2021

DOI: 10.1039/d1an00272d rsc.li/analyst structure or smell and taste should remain stable as long as possible.

A straight-forward method for studying photodegradation is irradiation of a solution of the sample in a vial or cell for a fixed time, followed by analysis of solution aliquots by e.g. liquid chromatography (LC). Using such an approach, for example, Confortin studied the degradation of the dye crystal violet (CV), Toohey investigated the effect of iron in presence of UV light on the sweetener rebaudioside A, and Hora examined the degradation of pharmaceutical compounds in wastewater. ${ }^{1-3}$ Similar approaches have been applied to e.g., pharmaceuticals, ${ }^{4-9}$ pesticides, ${ }^{10,11}$ foodstuffs, ${ }^{12}$ and polymers. $^{13,14}$

These approaches mostly work well if a single, fairly pure compound is studied and a limited number of degradation products is formed. However, if the sample is a (complex) mixture and potentially contains unknown components, interpretation of the results becomes very difficult. This complexity increases when multiple types of molecules degrade and/or start to react with other sample components or formed fragments. A major challenge in such photodegradation studies is, therefore, to distinguish which degradation products arise from which (parent) molecule.

A way to decrease sample complexities that are met in LID studies, might be to carry out a separation of the sample components prior to light exposure. This preferably would be done 
in an on-line fashion where a separation technique like LC is coupled to a low-volume irradiation cell in which isolated components can be exposed to light. Ideally, such a cell would also allow real-time spectroscopic monitoring of the content. After exposure, the cell content could be transferred on-line to another LC system for separation and analysis of the degradation products. On-line post-column photoreactors have been reported for reaction detection of target compounds, ${ }^{15-18}$ however, these reactors are less suited for on-line LID studies. The continuous flow reactors have relatively large volumes, the actual illumination of the sample is hard to control, and they do not allow in situ monitoring of photochemical reactions.

Liquid core waveguides (LCWs) are tubes of small internal diameter that are based on the principle of total internal reflection (TIR) and are typically made of low refractive index (RI) materials, which behave as an optical fibre once filled with a liquid of higher RI. They might offer a solution to the abovementioned challenges faced in on-line coupling of photoreactors. The possibility of coupling LCWs with LC has already been proven to be possible in earlier work where the LCW was used as a post-column (chemically-active) detection cell. $^{19-21}$ Therefore, we anticipate that on-line coupling of an LCW as an LID cell to LC in a pre- or post-column fashion will be feasible. LCWs facilitate axial illumination of its entire content and the possibility to detect the transmitted light, making the optical path length of an LCW quite significant (up to $1 \mathrm{~m}$ ), which is an advantage when concentrations of exposed components are low. Furthermore, since the light source is focused inside the capillary, the light dose can be easily controlled. Because of these properties, Teflon AF capillaries or coatings have been used for sensitive UV-Vis absorbance, ${ }^{22,23}$ fluorescence, ${ }^{24,25}$ mid-IR, ${ }^{26}$ and Raman spectroscopic detection. ${ }^{27-31}$

LCWs are typically made of Teflon AF, which is an amorphous and translucent polymer of tetrafluoroethylene (TFE) and 2,2-bis(trifluoromethyl)-4,5-difluoro-1,3-dioxole (PDD) with an RI of 1.29-1.31. This makes it suitable as an LCW when using core solvents such as water $(\mathrm{RI} \approx 1.33)$, methanol $(\mathrm{RI} \approx 1.333)$ and acetonitrile $(\mathrm{RI} \approx 1.34)$, which all are frequently used in LC eluents. There are currently two types of LCWs that are fabricated using Teflon AF. Type I is a tube entirely made of such a polymer, whereas type II is a silica capillary coated externally with Teflon AF. ${ }^{32}$ For a type II LCW, TIR occurs both at the core/silica and the silica/polymer interface, making it less suited as LID cell because a significant fraction of the light will travel through the capillary wall rather than through the liquid core where the sample will be located. For type I LCWs, TIR occurs at the core/polymer interface, providing optimal light-exposure of the total content in the LCW. An additional benefit of the use of type I LCWs made of Teflon AF is their permeability to gases, allowing diffusion of e.g. oxygen into the cell required for photooxidation processes. ${ }^{33,34}$

This study investigates the potential of a Teflon AF2400 LCW as an LID cell coupled to a spectrograph allowing efficient illumination together with collection of real-time transmission and absorption spectra of the cell content. The light transmission characteristics of LCWs of different length were studied as a function of wavelength. The effects of filling procedures and stability throughout the measurements were evaluated, and the diffusion of oxygen gas through the cell walls was verified using methylene blue as indicator. The absolute photon flux in the cell was determined by actinometry. The basic performance of the new LID cell was evaluated by monitoring the degradation of eosin Y (EY) and crystal violet $(\mathrm{CV})$ in the LCW and subsequent analysis of the content of the LID by LC. To the best of our knowledge, the use of an LCW cell for studying photodegradation has not been reported before.

\section{Materials and methods}

\subsection{Chemicals}

For the preparation of LC eluents, Ultrapure Milli-Q water (MQ), methanol (MeOH) (Biosolve, UPLC/MS grade), formic acid (FA) (Sigma-Aldrich), sodium hydroxide (NaOH) (SigmaAldrich) and triethylamine (TEA) (Sigma-Aldrich) were used. Actinometry experiments were performed using 1,2-bis(2,4dimethyl-5-phenyl-3-thienyl)-3,3,4,4,5,5-hexafluoro-1-cyclopentene (DAE) obtained from TCI Chemicals (Tokyo, Japan) dissolved in hexane (Sigma-Aldrich). In order to assess gas diffusion, methylene blue hydrate (Sigma-Aldrich, $\geq 97 \%$ purity), $\mathrm{NaOH}$ and glucose (Sigma) were used. Degradation experiments were executed using solutions of eosin Y (SigmaAldrich, $\geq 99 \%$ purity) and crystal violet (Sigma-Aldrich, $\geq 90 \%$ purity) dissolved in MQ.

\subsection{LID cell set-up and detection system}

A schematic overview of the LID set-up is shown in Fig. 1. Illumination was performed with a Xenon lamp purchased

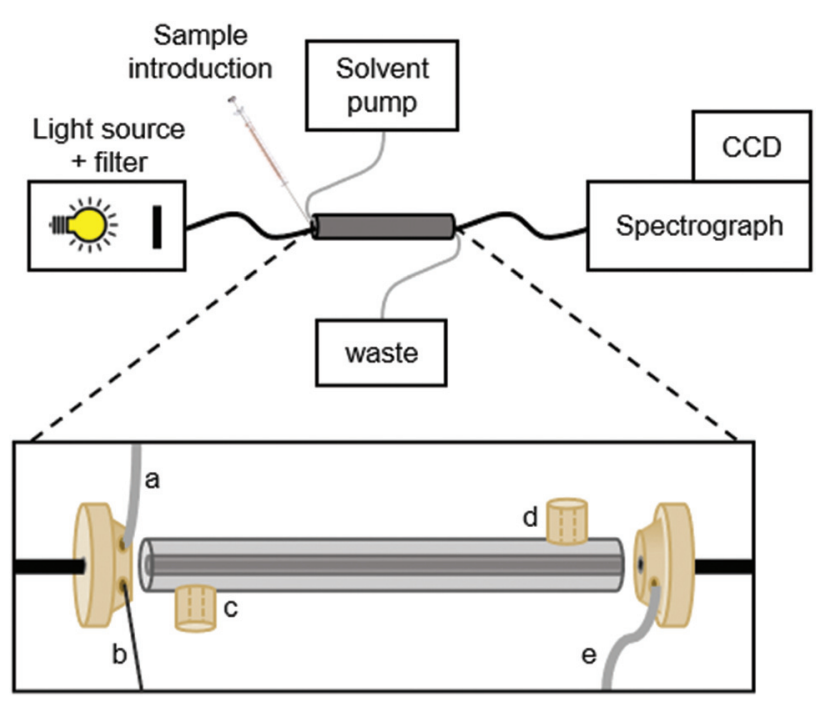

Fig. 1 Schematic overview of the design of the LID cell. From left to right: (a) connection with solvent pump, (b) sample delivery by syringe, (c) gas inlet, (d) gas outlet, (e) waste channel. 
from Hamamatsu. When only a specific part of the spectrum of the Xenon lamp was required, bandpass filters were placed inside the lamp housing. The light from the lamp was guided through an optical fibre (core diameter, $1500 \mu \mathrm{m}$ ) into the LID cell via a coupling piece (Fig. A.1, ESI†). An identical coupling piece was used at the end of the LID cell to measure the transmitted light. The coupling pieces were made of PEEK and designed with three threaded inlets: one in the axial face for fitting the LID cell, and two side inlets at a $45^{\circ}$ angle. The back of the pieces were sealed with a quartz window to allow introduction or collection of light. The two side inlets at the first coupling piece can be used for introduction of the sample and (if necessary) another solvent or reagent e.g., a catalyst or inhibitor; details of sample introduction are provided in section 2.3. The side inlets of the coupling piece at the end of the LCW were used for waste or for sample collection.

The LID cell consisted of a type I LCW made of Teflon AF2400 (ID, $800 \mu \mathrm{m}$; OD, $1000 \mu \mathrm{m}$ ), obtained from Cambridge Reactor Design (Cottenham, United Kingdom), which was placed inside an outer tubing made of high-density polyethylene (HDPE), creating a tube-in-tube design. T-pieces made of PEEK were glued into the outer tubing to permit addition of gases, such as nitrogen or oxygen (see Fig. 1). Both ends of the LID cell were glued into standard chromatographic finger-tight fittings with Valco thread facilitating the connection of the LID cell to the coupling pieces. To study the effect of gas permeability of the type I LCW, type II LCWs were also used which are not gas permeable. These were made of fused-silica, coated externally with $\pm 15 \mu \mathrm{m}$ Teflon AF2400 (ID, $100 \mu \mathrm{m}$; OD, $363 \mu \mathrm{m}$ ), obtained from CM Scientific Ltd (Silsden, United Kingdom). The type II LCW was fitted with two chromatographic finger-tight fittings using micro sleeves to prevent leaking.

Transmitted light was collected by use of an optical fibre at the other end of the cell that was connected to an SR-163 spectrograph equipped with a DV420A CCD camera, both from Andor Technologies (Belfast, UK). The distance of the optical fibre from the cell could be varied to either increase or decrease the intensity of the measured signal. Generally, a $1 \%$ transmission filter was placed in between the end of the LID cell and the optical fibre to reduce the signal and to avoid overloading the detector response. The spectrograph was equipped with a grating of 600 lines per $\mathrm{mm}$ blazed at $300 \mathrm{~nm}$, a light entrance slit with adjustable width, and a back illuminated CCD chip of $256 \times 1024$ pixels with a pixel size of $26 \mu^{2}$ for detection of the dispersed transmitted light. The spectral range covered by the CCD camera in non-scanning mode was about $263 \mathrm{~nm}$ and was calibrated using a $\mathrm{Hg}$ calibration lamp. The CCD chip was cooled at $-60{ }^{\circ} \mathrm{C}$. Unless stated differently, detection settings used for transmission measurements were: slit width, $10 \mu \mathrm{m}$; exposure time, $0.1 \mathrm{~s}$; vertical shift speed, $8.25 \mu \mathrm{s}$; horizontal read-out, $33 \mathrm{kHz}$ at 16-bit. Acquired spectra were collected using the Full Vertical Binning mode and were background corrected in the case of absorption measurements. Data acquisition was performed using Solis software from Andor Technologies.

\subsection{Injection/filling}

The LID cells were filled and flushed with solvent by means of a K-500 solvent pump (Knauer, Berlin, Germany) at a flow rate of $200 \mu \mathrm{L} \mathrm{min}{ }^{-1}$. The pump was connected to one of the side inlets of the first coupling piece, unless specified otherwise. Samples of EY and CV were directly introduced using a syringe, which was connected to the second inlet, while the solvent pump was turned off. The cell was cleaned in between samples by flushing with $300 \mu \mathrm{L}$ of $\mathrm{MeOH}$ followed by $300 \mu \mathrm{L}$ of MQ using the solvent pump.

\subsection{LC system}

In order to prepare the LC eluent, a buffer was made by adding $\mathrm{FA}$ and $\mathrm{NaOH}$ to $1 \mathrm{~L}$ of $\mathrm{MQ}$ at a final concentration of $0.1 \mathrm{M}$ and $0.02 \mathrm{M}$ respectively, to obtain a $\mathrm{pH}$ of 3 . The eluent was prepared by mixing buffer and $\mathrm{MeOH}$ in ratios of 95/5 [v/v] respectively for mobile phase $\mathrm{A}$, and $5 / 95[\mathrm{v} / \mathrm{v}]$ for $\mathrm{B}$. TEA was added as an ion pairing agent to both $\mathrm{A}$ and $\mathrm{B}$ at a final concentration of $5 \mathrm{mM}$. All analyses were carried out on an Agilent 1100 series LC system equipped with a quaternary solvent delivery system, a column oven, an autosampler and a photodiode array detector (PDA). A ZORBAX Eclipse RRHD C18 column $(2.1 \times 150 \mathrm{~mm}$; particle size, $1.8 \mu \mathrm{m})$ and a security guard column $(2.1 \times 5 \mathrm{~mm})$ containing the same C18 packing were both obtained from Agilent. Gradient elution was performed going linearly from $5 \%$ to $95 \%$ B in $10 \mathrm{~min}$. After $5 \mathrm{~min}$ at $95 \% \mathrm{~B}$, the mobile phase changed back to $5 \%$ B. Including $15 \mathrm{~min}$ of equilibration time, the total analysis time was $30 \mathrm{~min}$. The flow rate was set at $150 \mu \mathrm{L} \mathrm{min} \operatorname{mon}^{-1}$ and the column oven at $40{ }^{\circ} \mathrm{C}$. Spectral data were recorded from 250 to $800 \mathrm{~nm}$ with a resolution of $1.0 \mathrm{~nm}$, a sampling rate of $144 \mathrm{~Hz}$, and analysis monitoring was set at detection the wavelengths 254, 400, 515, 550, and $595 \mathrm{~nm}$. The equipment was controlled by ChemStation Chromatography Data Software from Agilent.

\section{Results \& discussion}

The performance of the LCW is first characterized with respect to parameters affecting its transmittance. We then evaluate the photon flux and gas permeability of the Teflon AF2400 LCW. Finally, we demonstrate the applicability of the LCW in a proof-of-concept experiment studying the light-induced degradation of EY and CV.

\subsection{LCW transmittance}

The light transmittance of the LCW inside the LID cell is a critical property: it determines both the photodegradation rate as well as the detection limit when used in combination with spectroscopy. Preferably, light losses should be minimized to achieve a consistent illumination of the entire solution. Transmittance of the LCW can be dependent on, or influenced by several parameters, such as length of the LCW, concentration and molar absorption coefficient of the sample components, scattering losses inside the LCW, pressure applied on 
the LCW, and temperature. Furthermore, it has recently been reported that when transmittance is sufficient, small internal LCW diameters $(<1 \mathrm{~mm})$ allow higher and a more homogeneous irradiance resulting in shorter reaction times of photochemical reactions. ${ }^{35}$ For this study we used LCWs with an ID of $800 \mu \mathrm{m}$ and the effects of the above-mentioned parameters were studied.

3.1.1 Length of LCW. The use of an LCW as LID cell allows exposure of the entire sample and the increased optical path length results in a higher overall absorbance, enhancing photodegradation efficiency. ${ }^{31}$ However, the length of the LCW should be chosen carefully, as light transmittance is expected to decrease exponentially with the optical path length when an absorbing compound is present. Overall absorbance should not be too high, as light should still reach the detector to obtain spectral information and molecules at the end of the LCW should still be exposed sufficiently.

The influence of the cell length was tested by fabricating four LID cells using type I LCWs of $12 \mathrm{~cm}$ (cell volume $\sim 60 \mu \mathrm{L}), 20 \mathrm{~cm}(\sim 100 \mu \mathrm{L}), 40 \mathrm{~cm}(\sim 200 \mu \mathrm{L})$ and $60 \mathrm{~cm}$ $(\sim 300 \mu \mathrm{L})$ long.

These were filled with water and the intensity of the transmitted light was measured for each cell at different wavelengths using specific bandpass filters. The intensities followed from power measurements in triplicate (each time after realignment, $\mathrm{RSD}<9 \%$ ), where the sensor of the power meter was positioned at the excitation fibre outlet or at the exit of the LCW filled with MQ. The light transmittance was calculated by comparing the intensity at the end of the LCW $(I)$ with the intensity at the exit of the excitation fibre $\left(I_{0}\right)$. Fig. 2 shows the average $(n=3)$ transmittance of the four LCWs at different wavelengths, normalized to the highest transmittance value measured for the $12 \mathrm{~cm} \mathrm{LCW}$ at $630 \mathrm{~nm}$. Fig. 2a shows that the transmittance decreases significantly with increasing length. Light losses are most probably due to reflection losses caused by pores and imperfections in the Teflon capillary surface, as also indicated by Altkorn et $a .^{31}$ The number of reflections increases with the length of the capillary, causing more light to be lost. Apparently, this effect is strongest in the first part of the LCW; the difference between LCWs of 40 and $60 \mathrm{~cm}$ was found to be negligible in our experiment.

The light absorbance by a compound inside the cell is a point of consideration. Increased analytical sensitivity due to a long optical path length might be beneficial, however, from the viewpoint of analyte degradation, the formation of a strong light-intensity gradient inside the LID cell is not favoured. Therefore, for LID studies of samples showing significant light absorbance at the applied wavelength, medium-length LCWs are preferred over very long ones. Another reason to limit the length of the LCWs, is to increase compatibility with LC. LCW volumes larger than LC peak volumes would cause loss of LC resolution when using the cell in a post-column setup.

3.1.2 Wavelength of light. The LID cell should ideally provide adequate light transmittance over a broad wavelength range. Its wavelength dependency was tested by measuring the light transmittance of an LCW filled with MQ using bandpass filters of 450, 500, 532, 580 and $633 \mathrm{~nm}$ (nominal values) (Fig. 2b). The LCW can be used over this entire range, but the transmittance increases with increasing wavelength. The porosity and imperfections of the polymer surface might cause light losses by scattering, which will be stronger for photons of shorter wavelength. The observed wavelength dependencies of the light transmittance, although not very large, should be considered when using polychromatic light sources for photodegradation.
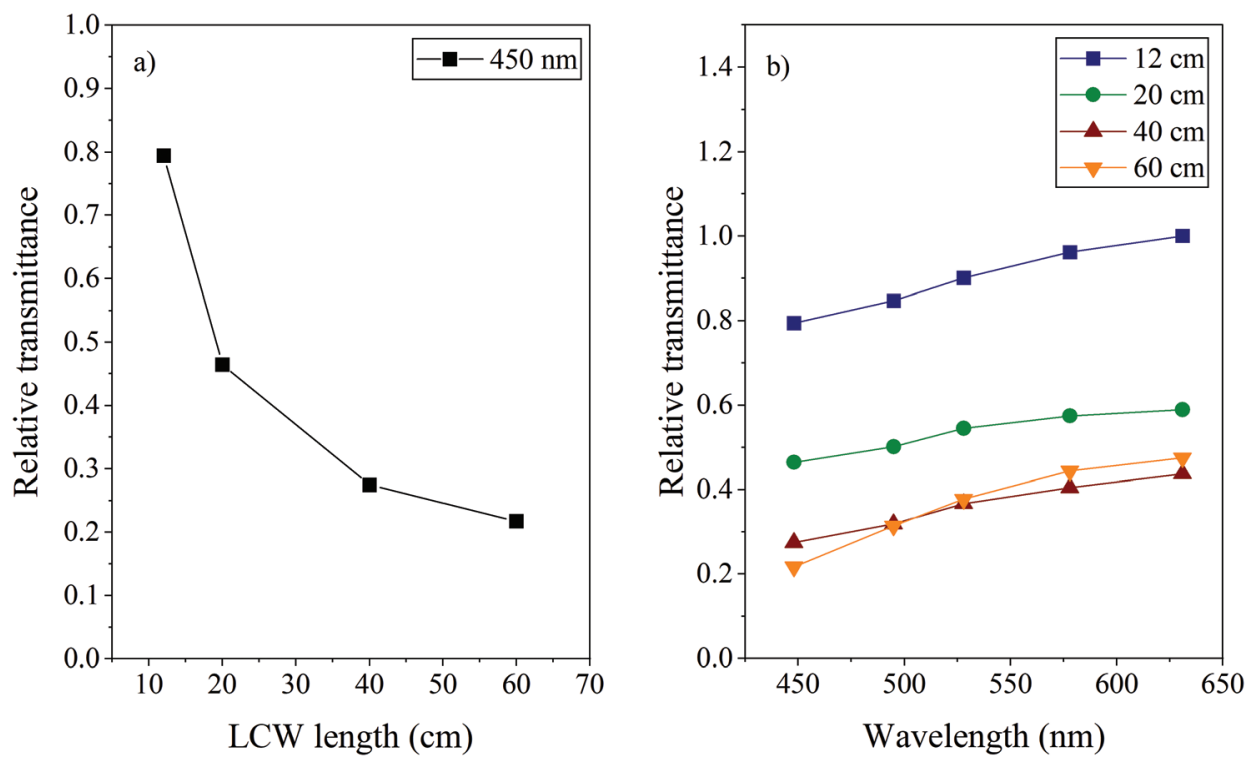

Fig. 2 (a) The relative transmittance for light of $450 \mathrm{~nm}$ as function of LCW length calculated by comparing the transmitted (I) with the input power $\left(I_{0}\right)$; (b) the relative transmittance for light of different wavelengths using LCW lengths of $12,20,40$ and $60 \mathrm{~cm}$. 
3.1.3 Variation due to filling procedure. The performance of Teflon AF2400 cells, e.g. for absorbance spectroscopy, might be sensitive to changes in liquid pressure caused by solvent injection or filling of the cell. ${ }^{36}$ Therefore, the light throughput of the LID cell was measured after filling a type I LCW in triplicate using an HPLC pump at flow rates of 50, 100, 150, 200 and $250 \mu \mathrm{L} \mathrm{min}{ }^{-1}$. The average transmitted light intensities with corresponding spread (standard error) are shown in Fig. B.1 (left) in the ESI. $\uparrow$ The average measured intensities at the applied flow rates did not differ significantly. The standard error, however, decreased going from 50 up to $200 \mu \mathrm{L} \mathrm{min}{ }^{-1}$, but increased again at $250 \mu \mathrm{L} \mathrm{min}{ }^{-1}$. It is hypothesized that flow rate and pressure may affect to what extent the liquid penetrates air-filled pores in the Teflon material and this would affect the TIR properties of the LCW. ${ }^{37}$ We also observed that filling the LCW manually by syringe results in variability of the transmittance, which is most likely caused by irregularities in the applied pressure. When the same experiment was performed using a type II LCW, the effect of different flow rates on transmittance and spread was negligible (Fig. B.1, $\dagger$ right). Therefore, we conclude that the porous Teflon AF wall is more susceptible to pressure fluctuations and that it is important to choose a well-reproducible filling or injection method using a stable pump which transfers the sample from the autosampler to the LID cell.

3.1.4 Signal stability. During a typical photodegradation experiment, the LID cell is irradiated for a prolonged period of time, while simultaneously spectral data are collected. To test the cell stability, repetitive light throughput measurements of $1 \mathrm{~min}$ each over a period of $90 \mathrm{~min}$ were performed in triplicate with a type I and a type II LCW, both filled with MQ. Fig. 3 shows the measured intensities over time for the LCWs. The graph for the type I LCW shows a quick rise of the intensity after filling of the cell and a more or less constant signal after 5 min. For the type II, however, it takes (much) longer for the signal to stabilize and the variation between measurements is larger. A reason for this difference may be found in the external silica coating of the type II modified Teflon. Teflon AF2400, like other polymers, is susceptible to temperature changes, as Dlubek et al. ${ }^{37}$ described. Increased temperature results in an increased hole-free volume, which lowers the RI of the LCW and may affect transmittance. We hypothesize that due to the silica coating in the type II LCW, heat may not be exchanged efficiently and a slow increase in transmittance is observed. For the type I LCW, the thin wall and/or the permeability may contribute to better heat dissipation, providing stable signals after $5 \mathrm{~min}$ and allowing spectral data to be measured in a repeatable manner.

\subsection{Light absorption in LCW}

3.2.1 Absorbance as a function of analyte concentration. The LCW can be used as an absorbance cell allowing sensitive, in situ monitoring of photodegradation. The performance of the LCW for absorption detection was determined by measuring the absorbance of an aqueous dilution series of CV. The absorbance at $585 \mathrm{~nm}$ of the solutions was plotted against the CV concentration (Fig. 4). The $12 \mathrm{~cm}$ LCW shows a clearly increasing absorbance over a relevant concentration range.

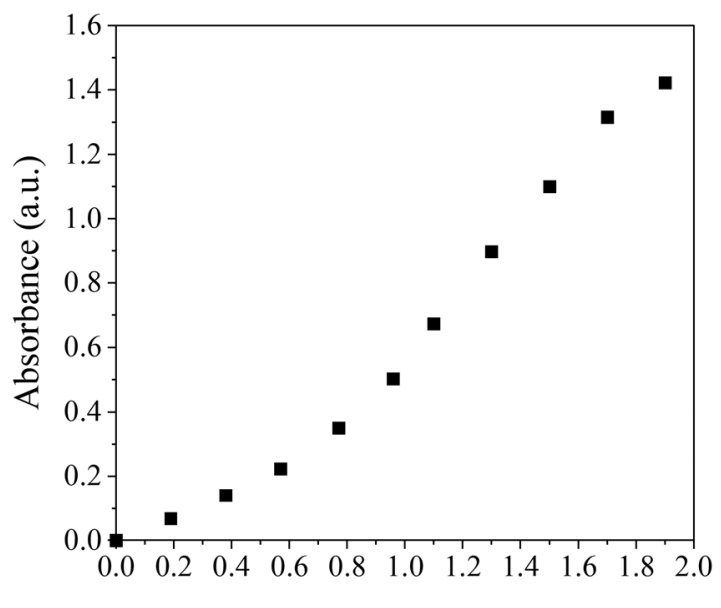

Concentration $(\mu \mathrm{mol} / \mathrm{L})$

Fig. 4 Measured absorbance as function of the concentration of CV measured in a $12 \mathrm{~cm}$ long LCW.
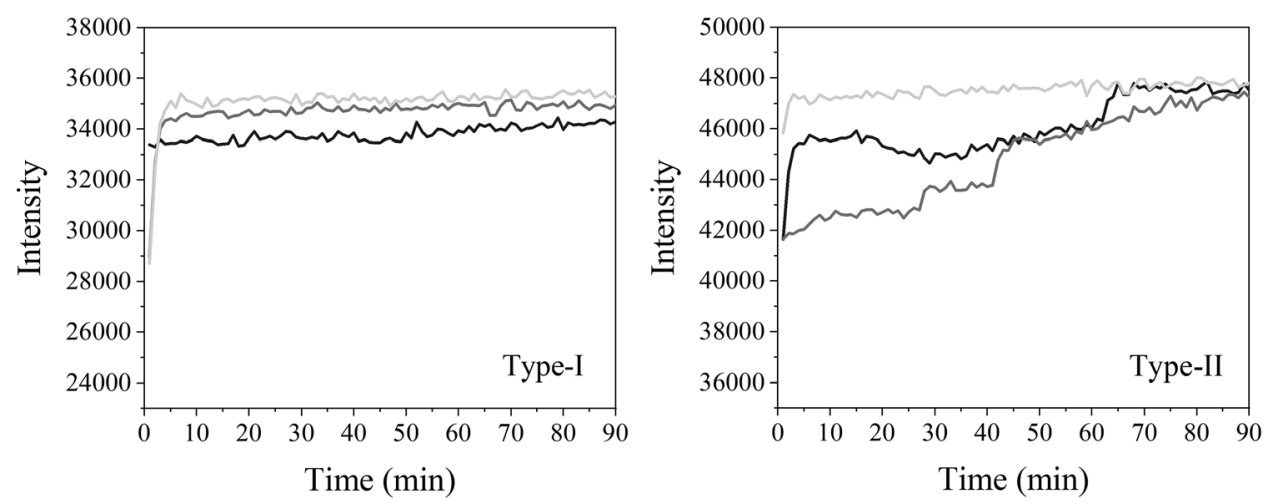

Fig. 3 The intensity of the transmitted light measured in triplicate at $462 \mathrm{~nm}$ every minute for 90 min for a type I LCW and a type II LCW filled with MQ. 

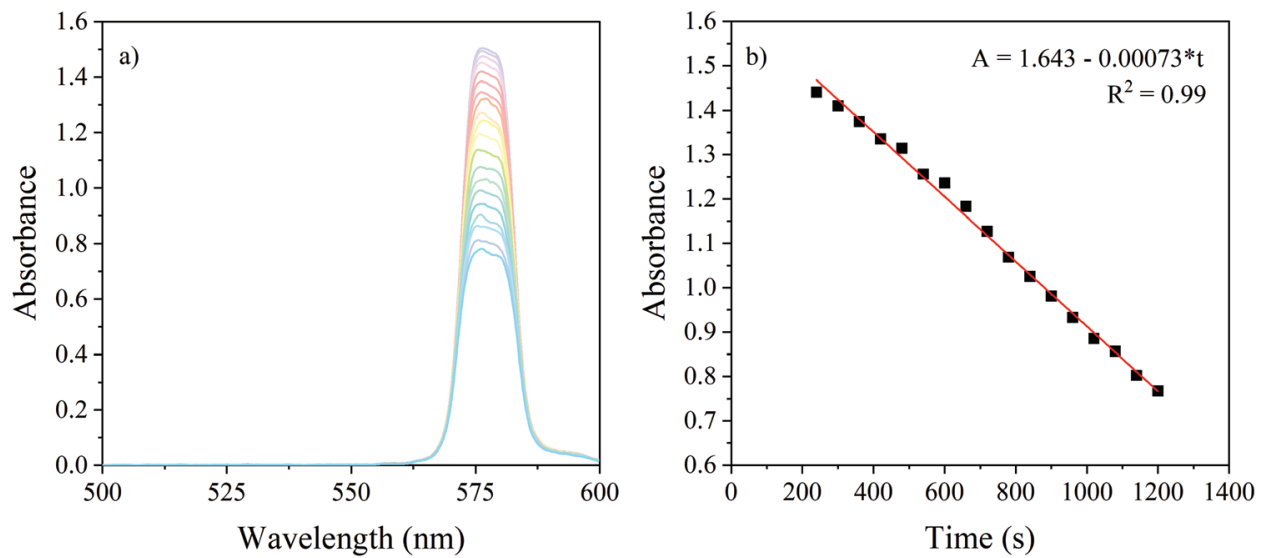

Fig. 5 Actinometry of the LCW. (a) Measured change in absorbance in real-time during the isomerization reaction of DAE-c to DAE-o at $578 \mathrm{~nm}$ inside a $20 \mathrm{~cm}$ long LID cell. (b) Linear part of the decrease in absorbance is plotted; slope was used to determine the photon flux (ESI $\dagger$ ).

Although not perfectly linear, the observed relation will enable us to correlate absorbance changes to changes in molecular composition of the sample due to photodegradation. Notably, the design of the LCW LID cell is very suitable for measuring low concentrations, for instance after sample pre-separation with LC, where the concentration of minor components can be rather low.

3.2.2 Photon flux in LCW determined by actinometry. In order to assess photodegradation processes, the photon flux (photons/sec) inside the LID cell should be known. This was determined using a chemical actinometer: a compound that undergoes a light-induced reaction for which the quantum yield $(Q)$ is known. The actinometer of choice was DAE, a diarylethene derivate known for its photoisomerization reaction. The colour change of DAE between violet and colourless is a result of a ring-closing and -opening reaction, which takes place upon irradiation with either UV or Vis light and is reversible up to 3000 times (Fig. C. $1 \dagger$ ). ${ }^{38-40}$ This compound is especially suitable as an actinometer in closed systems, such as the LID cell, as the change in the absorption spectrum can be measured in real-time, so that sampling of the solution is not required. Furthermore, in contrast to e.g., ferrioxalate actinometers, DEA remains in solution throughout the reaction, making it compatible in combination with microchannels, such as the LCW.

A $20 \mathrm{~cm}$ LCW was filled with a solution of DAE in hexane and was irradiated with the Xenon lamp. Since this is a polychromatic light source, an equilibrium between the open and closed form will be reached after a certain period of time. Therefore, a UV shortpass filter $(<350 \mathrm{~nm})$ was used to turn DAE into the violet closed form (DAE-c), and a bandpass filter of (nominal value) $580 \mathrm{~nm}$ was used to allow DAE to turn into the colourless open form (DAE-o). This wavelength was chosen because it is close to the absorption maximum of DAE-c. The actual maximum transmitted wavelength of the bandpass filter was determined to be $578 \mathrm{~nm}$ and was taken into account. Calculations and technical aspects regarding the measured photon flux are further elaborated in the ESI. $\dagger$
Fig. 5 shows the decrease in absorbance in real-time for the reaction from DAE-c to DAE-o at $578 \mathrm{~nm}$. The linear part of the decrease in absorption was plotted and used to calculate the average rate of the conversion. From this, the photon flux at $578 \mathrm{~nm}$ was calculated. This was compared to the flux calculated from power measurements, obtained by placing the power meter behind the end of the LID cell filled with water. Using actinometry, a photon flux of $10.3 \times 10^{12}$ photons per $\mathrm{s}$ was calculated, compared to $9.49 \times 10^{12}$ photons per $\mathrm{s}$ obtained from power measurements. The similarity between the obtained results with both methods indicate that the power measurements are a good estimate of the photon flux passing the LCW. However, the power meter measures the number of photons reaching the end of the LID cell, whereas the actinometer measures the effective intensity inside the LCW. Therefore, we presume that the DAE actinometer gives a better representation of the real photon flux inside the LID cell.

\subsection{Gas diffusion through LCW wall}

Oxygen gas plays an important role in many photodegradation reactions. The Teflon AF LCW comprises a gas permeable wall, which offers the possibility to influence the photodegradation process by adding or removing oxygen gas to or from the exposed solution. Oxygen diffusion was tested by using methylene blue $(\mathrm{MB})$ as an indicator for oxygen. The LID cell was filled with an aqueous solution of $\mathrm{MB}$, glucose and sodium hydroxide. In the presence of oxygen, $\mathrm{MB}$ is oxidized and the solution will turn blue, resulting in absorption of visible light by MB. When oxygen is removed or consumed, MB will be reduced by the sodium hydroxide and the solution turns colourless, resulting in a reduced visible absorbance. The decolourization reaction of $\mathrm{MB}$ by oxygen was recorded in real-time by measuring the transmitted light intensity of the LCW at the absorption maximum of MB (668 nm).

Fig. 6 shows the LCW light transmittance in real-time (yellow curve) while the outer tubing of the LID cell, which initially contained air, was flushed with nitrogen gas. During 


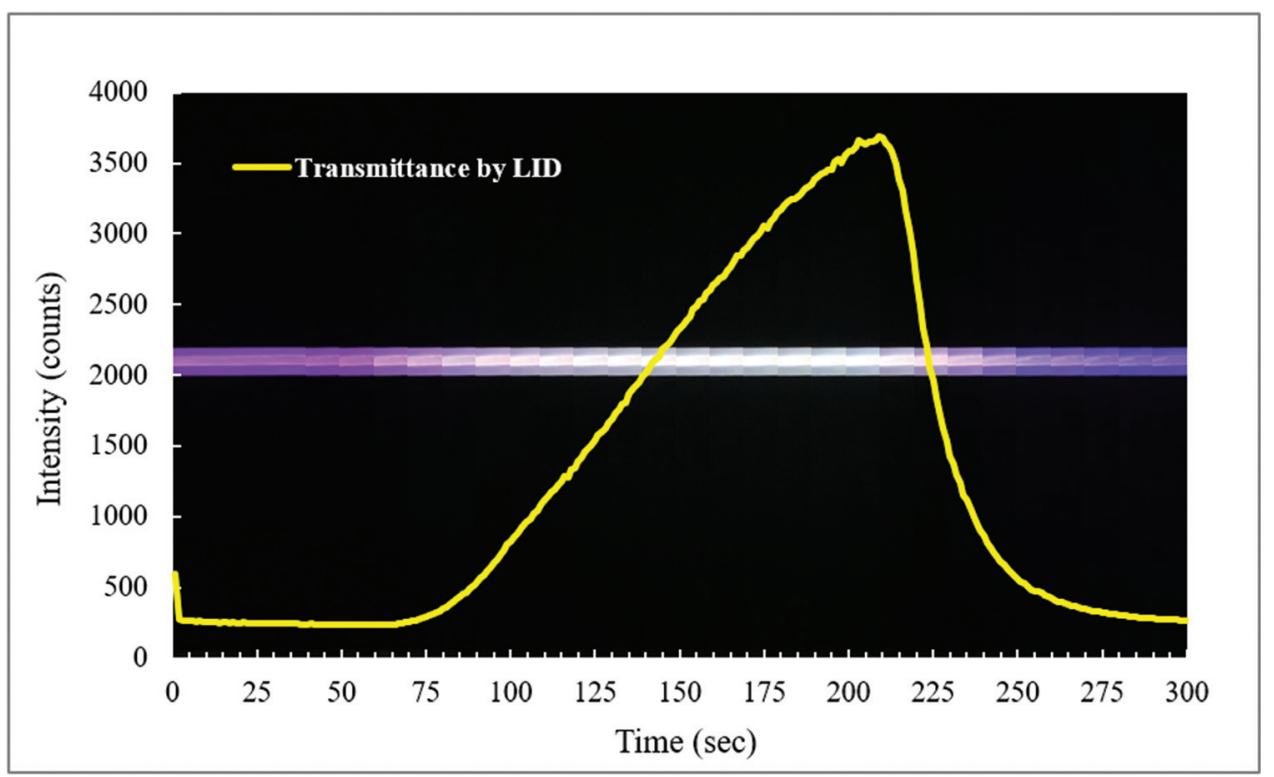

Fig. 6 Monitoring of oxygen in the LCW. The LID cell was filled with a solution of MB, glucose and $\mathrm{NaOH}$ which was used as reagents to observe the presence of dissolved oxygen in the LCW. The yellow curve shows the measured transmittance of the LCW at $668 \mathrm{~nm}$. The blueish trace shows the actual colour of the LCW at the corresponding time points. When the oxygen concentration decreases by flushing nitrogen gas through the outer tubing (50-200 s), MB is reduced and turns colourless.

the flushing, part of the LID cell was filmed using a camera and fragments of this footage are shown at the corresponding time points. After about $50 \mathrm{~s}$, the nitrogen flow was turned on, and from $65 \mathrm{~s}$ onwards the transmitted light intensity increased, indicating decolourization, thus reduction of $\mathrm{MB}$ caused by oxygen depletion in the LCW. After about $200 \mathrm{~s}$ the nitrogen flow was turned off, and subsequently a reduction of the transmittance was observed, implying oxidation of MB due to presence of oxygen in the LCW. The latter means that spontaneous diffusion of oxygen occurs from air back into the solution. Since the solution inside the LCW is not stirred, we assume an oxygen gradient will exist from the wall to the middle of the LCW. As expected, the video recording of the colour changes showed no sign of inhomogeneity along the length dimension of the LCW. This experiment showed that the Teflon AF wall is gas permeable even at ambient pressure, which is in line with previous findings. ${ }^{41}$ The experiment also demonstrated that oxygen levels in the LCW can be controlled using the tube-in-tube design, and that the reaction process can be followed in real-time by absorbance spectroscopy. This is a clear advantage of the type I LCW over the type II, as it allows the study of photodegradation under oxygen-saturated conditions and evaluation of the effect of oxygen on degradation, providing more detailed mechanistic insight.

\subsection{Monitoring the LID of EY and CV}

Two proof-of-concept experiments were performed by monitoring the photodegradation of the dyes EY (Fig. D.1 $\dagger$ ) and CV (Fig. D. $2 \dagger$ ). The photodegradation processes of these dyes are well known, allowing thorough evaluation of the results obtained with the LCW setup and LC analyses. A $12 \mathrm{~cm} \mathrm{LCW}$ was filled with a $5 \times 10^{-7} \mathrm{M}$ solution of $\mathrm{EY}(\varepsilon$ at $518 \mathrm{~nm}$, $95000 \mathrm{~cm}^{-1} \mathrm{M}^{-1}$ ), and subsequently exposed to light from the Xenon lamp for $3 \mathrm{hrs}$ while recording absorption spectra in real time every $10 \mathrm{~min}$. An aliquot of the starting (unexposed) EY solution was also analysed by LC with PDA detection. After $3 \mathrm{hrs}$ the exposed EY solution was collected off-line by flushing the LID cell with $60 \mu \mathrm{L}$ of $\mathrm{MeOH}$. The collected sample was immediately analysed by LC-PDA in order to measure potential degradation products. The same procedure was followed for a $2.5 \times 10^{-6} \mathrm{M}$ solution of $\mathrm{CV}\left(\varepsilon\right.$ at $\left.590 \mathrm{~nm}, 87000 \mathrm{~cm}^{-1} \mathrm{M}^{-1}\right)$, but then using a $5 \mathrm{hr}$ light exposure time.

3.4.1 Eosin Y. According to Alvarez-Martin et al., the degradation of EY in the presence of oxygen follows an oxidative process resulting in breakdown of the chromophore and consequently full discolouration. ${ }^{42}$ Under anoxic conditions, debromination of EY takes place while its chromophore remains intact. Fig. 7a shows the in situ absorption spectra recorded during the 3-hrs irradiation of EY. A clear and steady decrease of the absorbance in time over the whole visible EY spectrum was observed, indicating a loss of the chromophoric moiety by light degradation of the EY present in the LCW. During $3 \mathrm{hrs}$ light exposure, the absorbance intensity of $\mathrm{EY}$ at $515 \mathrm{~nm}$ reduced with $97 \%$. The LC chromatograms recorded at $515 \mathrm{~nm}$ obtained before and after light exposure of the EY solution are shown in Fig. $7 \mathrm{~b}$ with EY eluting at $18.4 \mathrm{~min}$. The peak area obtained for $\mathrm{EY}$ in the light-exposed solution is only $4 \%$ from the EY peak area of the solution before irradiation, which correlates nicely with the reduction in absorbance observed in the LCW measurements. For EY, debromination will take place in the first phase of degradation. Since the UV/Vis absorption spectra of partly debrominated $\mathrm{EY}$ are similar to that of EY, ${ }^{43}$ 

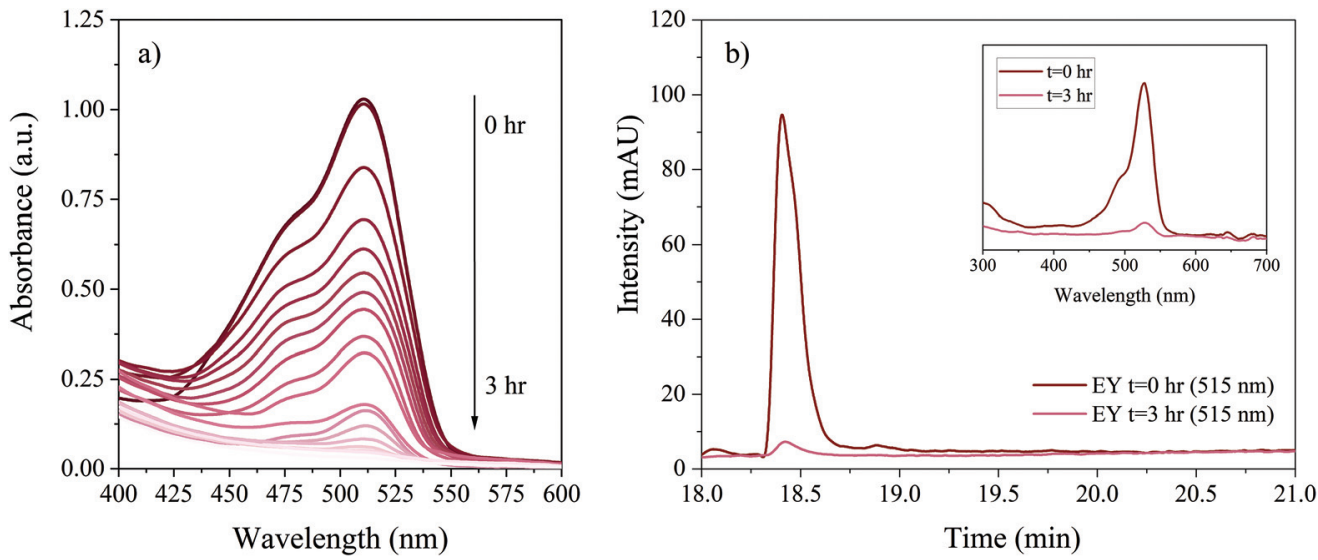

Fig. 7 (a) Real-time absorption spectra of a solution of EY $\left(5 \times 10^{-7} \mathrm{M}\right.$ in water) present in the LID-cell during light irradiation for 3 hrs; (b) LC chromatograms of the EY solution before (at $t=0 \mathrm{hr}$ ) and after exposure (at $t=3 \mathrm{hr}$ ) in the LID cell. The insert shows the PDA spectra obtained during LC analysis at $t=0 \mathrm{hr}$ and $t=3 \mathrm{hr}$ for the peak at a retention time of $18.48 \mathrm{~min}$.

no shift in absorption is observed in the LCW. The final degradation products only absorb in the UV region, as a result a decrease in response of the signal of EY is observed in the LCW, whereas EY degraded for 3 hrs mainly shows UV-absorbing components in the chromatogram (Fig. D. $3 \uparrow$ ). The change in absorbance during irradiation as provided by the LCW setup can directly be correlated to the degree of photodegradation.

3.4.2 Crystal violet. LID of CV revealed a more complicated degradation pathway than observed for EY, exhibiting both colourless as well as visible-light absorbing degradation products. The real-time absorption spectra obtained during light exposure of CV in the LCW over a $5 \mathrm{hr}$ period (Fig. 8) show a $32 \%$ decrease in the absorbance of the sample, accompanied by a slight blueshift of the absorption maximum. This implies that the chemical composition of the sample is changing due to photodegradation. The absorbance measured in the LCW is

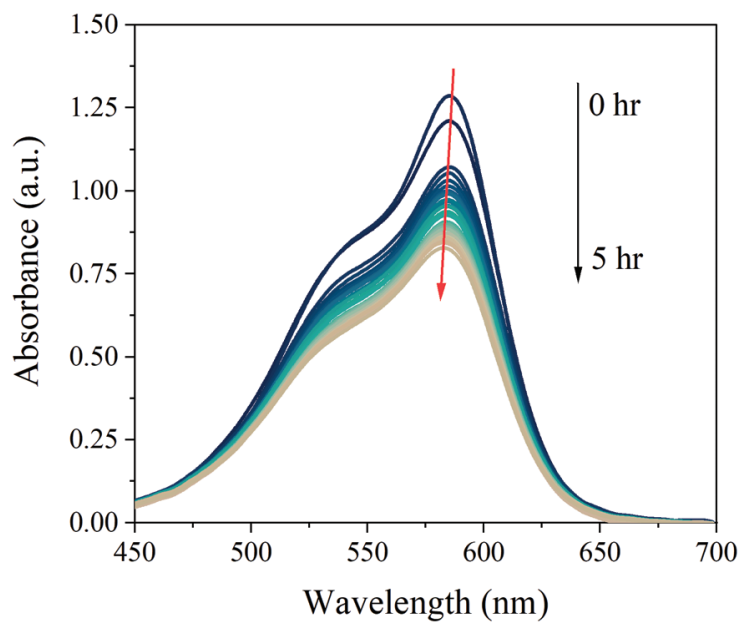

Fig. 8 Real-time absorption spectra of a solution of CV $\left(2.5 \times 10^{-6} \mathrm{M}\right)$ in water present in the LID cell during light irradiation for $5 \mathrm{hrs}$. the sum of the absorbance of all Vis-absorbing compounds (CV + degradation products). The absorption coefficients of the degradation products may differ from $\mathrm{CV}$ and are generally unknown. A decrease of $32 \%$ in absorbance suggests that apart from demethylation products (of which the chromophore is largely unaffected) a significant percentage of $\mathrm{CV}$ may have degraded into colourless products, such as dimethylaminobenzophenone (Michler's ketone) and dimethylaminophenol, also reported by Confortin and Kuramoto (Fig. D. $4 \dagger)^{1,44}$ LC-PDA analysis of the $5 \mathrm{hr}$ degraded CV sample showed several peaks $(11,12$ and 14) that exhibit absorption in the UV region. Of course, based on the absorption spectra only, the compounds could not be identified and additional MS analysis should be performed.

A slight shift towards shorter wavelength of the absorption maximum was observed for the whole sample during the in situ absorption measurements in Fig. 8. Evaluating the absorption spectra of the degradation products measured by the LC-PDA (Fig. D.5†), a similar blueshift was noticed for each degradation product, which was also observed in degradation research studies of $\mathrm{CV}$ by Confortin et al., and others. ${ }^{1,45,46}$ This is most likely a consequence of the demethylation process of $\mathrm{CV}$ resulting in a $5 \mathrm{~nm}$ shift of the absorption maximum for each newly formed product. Demethylation also results in a decreased retention time, caused by an increased polarity of the degradation products, which can be seen in Fig. 9. An overview of the detected analytes and tentative assignments is given in Table D.1. $\dagger$ When comparing the obtained results with those by Confortin $e t$ al. peaks 2,3 , and 4 are most likely mono-, di-, and tri-demethylated CV, respectively. Peak 5 may be an isomer of tri-demethylated $\mathrm{CV}$, and peaks 6 and 7 show similar absorption spectra as tetra-, and penta-demethylated CV. Three more peaks (8-10) were found of which peaks 8 and 9 show similar absorption maxima as that of diamond green B (Fig. D.6广). However, as Confortin also explains, these may also be assigned to $N$-oxide derivatives of CV. Peaks 11, 12 and 14 show absorbance in the 


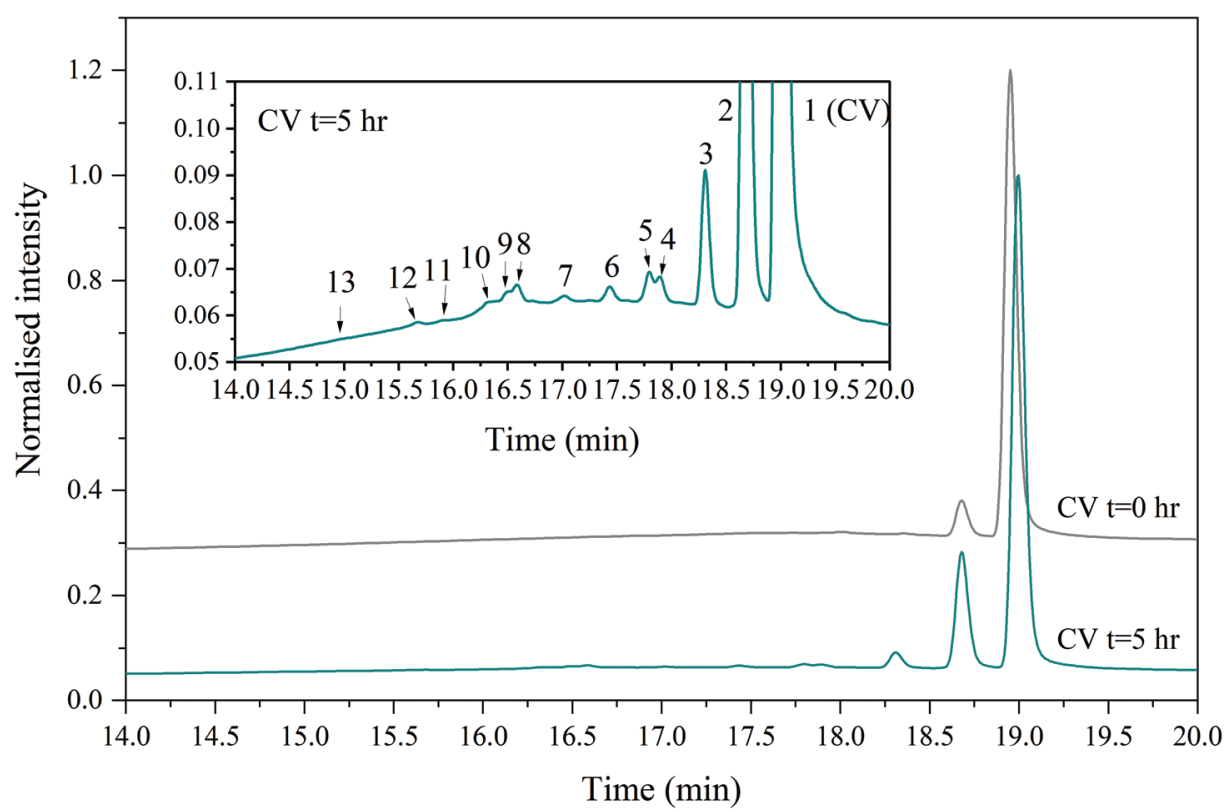

Fig. 9 Normalized LC-PDA chromatograms (at $595 \mathrm{~nm}$ ) of the CV solution before (at $t=0 \mathrm{hr}$ ) and after exposure (at $t=5 \mathrm{hr}$ ) in the LID cell. The insert shows a zoom of the chromatogram obtained at $t=5 \mathrm{hr}$ revealing the peaks of degradation products (2-13).

UV, but remain unidentified as the concentration was too low to obtain well defined absorption spectra and are therefore not shown. Peak 13, however, showed an absorption maximum of $546 \mathrm{~nm}$, which is similar to that of pararosaniline, which is completely demethylated CV. This was later confirmed by the analysis of a pararosaniline standard. The relative peak areas (derived from the LC-PDA signals) of CV and its degradation products, before and after irradiation, are shown in Fig. 10.

To make final conclusions about the identity of the abovementioned peaks, additional MS analyses should be per- formed. However, the most important result shown here is that a $>30 \%$ reduction of the main component $\mathrm{CV}$ was obtained after $5 \mathrm{hrs}$ of degradation. Weyermann and Confortin, who performed off-line degradation of $\mathrm{CV}$ in solution, reported near complete degradation after 25 and 115 hrs, respectively. ${ }^{1,45}$ For simple degradation processes, such as for $\mathrm{EY}$, the decrease in absorbance is a straightforward measure for the degradation rate. For compounds like CV, however, for which the degradation products also absorb in the same wavelength range as the parent compound, it is slightly more complicated to use the absorbance for this purpose.

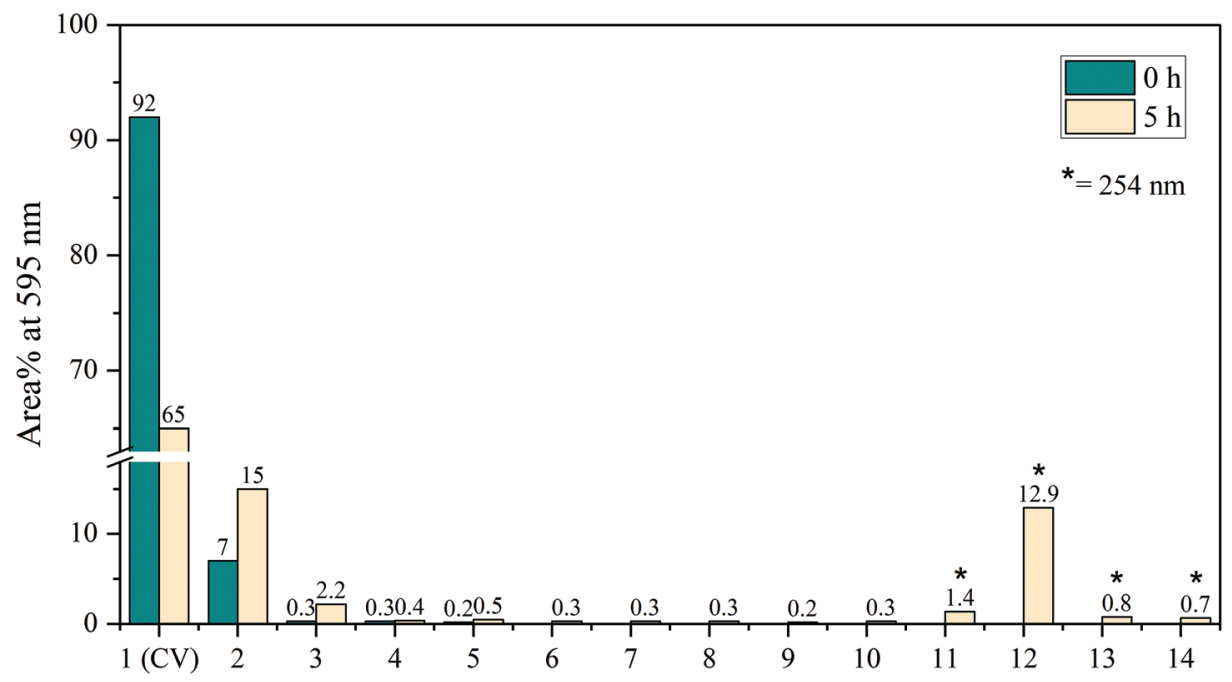

Fig. 10 Relative peak areas of CV and degradation products obtained by LC-PDA before ( $0 \mathrm{hr})$ and after (5 hr) exposure in the LID-cell. Peak areas are extracted at 595 or $254 \mathrm{~nm}\left(^{*}\right)$ if absorbance was in the UV. 


\section{Conclusions}

This study has shown that it is possible to initiate photodegradation of EY and CV while collecting real-time spectral data using an LID cell based on an LCW made of Teflon AF2400. The LCW is an efficient tool for illumination of the whole sample and is able to guide the light towards a detector. The results from the degradation experiments showed that realtime absorbance spectroscopy is useful for acquiring information on the stage of degradation. Changes in the shape of the absorption spectrum as well as the absorbance intensity are indicative for the extent of degradation. The results of the photodegradation of $\mathrm{EY}$ and $\mathrm{CV}$ are in line with previous studies, and the high photodegradation rate compared to conventional 'in-beaker' degradations looks promising. ${ }^{1,42,45,46}$ Clearly, the potential for on-line coupling of the cell to LC for further characterization of the degradants, comes as an advantage here.

There is also room for improvement. The speed of photodegradation could be enhanced by improving the efficiency of light coupling into the cell, and automation of the injection system may result in more reproducible spectral data as variation in applied pressure is reduced. The long path length of the LCW is beneficial for (diluted) samples with low absorbances. The transmittance by the LCW, however, decreases with the length of the capillary, caused by pores and imperfections in the material. As a result, the intensity of the light inside the LCW also decreases with length. Therefore, for LID studies, medium-length LCW are preferred over very long ones to prevent the formation of a strong light-intensity gradient inside the LID cell.

\section{Author contributions}

I. Groeneveld: conceptualization, methodology, investigation, resources, writing - original draft and editing; Suzan E. Schoemaker: methodology, investigation; Govert W. Somsen: writing - review and editing, supervision; Freek Ariese: writing - review and editing, supervision; Maarten R. Van Bommel: writing - review and editing, funding acquisition.

\section{Conflicts of interest}

The authors declare that there is no conflict of interests.

\section{Acknowledgements}

We thank the Precision Mechanics and Engineering group at the Vrije Universiteit Amsterdam, and Dick van Iperen in particular, for the close collaboration towards designing and building the LID cells. This work is part of the TooCOLD project (Toolbox for studying the Chemistry of Light-induced Degradation; project number 15506) carried out in the TTW
Open Technology Programme and is (partly) financed by the Netherlands Research Council (NWO).

\section{References}

1 D. Confortin, H. Neevel, M. Brustolon, L. Franco, A. J. Kettelarij, R. M. Williams and M. R. Van Bommel, J. Phys. Conf. Ser., 2010, 231, 1-9.

2 M. J. Toohey and L. N. Bell, J. Food Process. Preserv., 2019, 43, 1-5.

3 P. I. Hora, P. J. Novak and W. A. Arnold, Environ. Sci. Water Res., 2019, 5, 897-909.

4 M. Shamsipur, B. Hemmateenejad, N. J. Jahani and K. M. Majd, J. Photochem. Photobiol., A, 2015, 299, 62-68.

5 V. G. Bório, A. U. Fernandes and L. Silveira, Instrum. Sci. Technol., 2016, 44, 189-198.

6 R. Salgado, V. J. Pereira, G. Carvalho, R. Soeiro, V. Gaffney, C. Almeida, V. V. Cardoso, E. Ferreira, M. J. Benoliel, T. A. Ternes, A. Oehmen, M. A. M. Reis and J. P. Noronha, J. Hazard. Mater., 2013, 244-245, 516-527.

7 A. Y. C. Lin and M. Reinhard, Environ. Toxicol. Chem., 2005, 24, 1303-1309.

8 A. Y. C. Lin, X. H. Wang and W. N. Lee, Environ. Sci. Technol., 2013, 47, 4104-4112.

9 F. Gosetti, M. Bottaro, V. Gianotti, E. Mazzucco, P. Frascarolo, D. Zampieri, C. Oliveri, A. Viarengo and M. C. Gennaro, Environ. Pollut., 2010, 158, 592-598.

10 A. Kiss, S. Rapi and C. Csutorás, Microchem. J., 2007, 85, 13-20.

11 K. Sivagami, R. R. Krishna and T. Swaminathan, Sol. Energy, 2014, 103, 488-493.

12 V. T. Trang, Y. Kurogi, S. Katsuno, T. Shimamura and H. Ukeda, Int. Dairy J., 2008, 18, 344-348.

13 G. J. M. Fechine, P. A. Christensen, T. A. Egerton and J. R. White, Polym. Degrad. Stab., 2009, 94, 234-239.

14 E. Yousif, N. Salih and J. Salimon, J. Appl. Polym. Sci., 2011, 120, 2207-2214.

15 D. A. Volmer, J. Chromatogr. A, 1998, 794, 129-146.

16 P. J. Harman, G. L. Blackman and G. Phillipou, J. Chromatogr. B: Biomed. Sci. Appl., 1981, 225, 131-138.

17 S. X. Peng and S. M. Dansereau, J. Chromatogr. A, 2001, 914, 105-110.

18 T. Pérez-Ruiz, C. Martínez-Lozano and M. D. GarcíaMartínez, J. Pharm. Biomed. Anal., 2009, 50, 315-319.

19 A. F. Kadjo, P. K. Dasgupta and C. P. Shelor, Anal. Chem., 2020, 92, 6391-6400.

20 L. Ganranoo, S. K. Mishra, A. K. Azad, A. Shigihara, P. K. Dasgupta, Z. S. Breitbach, D. W. Armstrong, K. Grudpan and B. Rappenglueck, Anal. Chem., 2010, 82, 5838-5843.

21 D. Kottke, B. B. Burckhardt, J. Breitkreutz and B. Fischer, Talanta, 2021, 226, DOI: 10.1016/j.talanta.2021.122145.

22 K. Bescherer, J. A. Barnes and H. P. Loock, Anal. Chem., 2013, 85, 4328-4334. 
23 T. Rubles, D. Paige and C. Anastasio, Rev. Sci. Instrum., 2006, 77, 1-4.

24 Z. Liu and J. Pawliszyn, Anal. Chem., 2003, 75, 4887-4894.

25 A. Datta, I. Y. Eom, A. Dhar, P. Kuban, R. Manor, I. Ahmad, S. Gangopadhyay, T. Dallas, M. Holtz, H. Temkin and P. K. Dasgupta, IEEE Sens. J., 2003, 3, 788-795.

26 K. Flavin, H. Hughes and P. McLoughlin, Int. J. Environ. Anal. Chem., 2007, 87, 29-42.

27 B. J. Marquardt, P. G. Vahey, R. E. Synovec and L. W. Urgess, Anal. Chem., 1999, 71, 4808-4814.

28 S. Tanikkul, J. Jakmunee, M. Rayanakorn, K. Grudpan, B. J. Marquardt, G. M. Gross, B. J. Prazen, L. W. Burgess, G. D. Christian and R. E. Synovec, Talanta, 2003, 59, 809816.

29 R. J. Dijkstra, A. N. Bader, G. P. Hoornweg, U. A. T. Brinkman and C. Gooijer, Anal. Chem., 1999, 71, 4575-4579.

30 Y. Tian, L. Zhang, J. Zuo, Z. Li, S. Gao and G. Lu, Anal. Chim. Acta, 2007, 581, 154-158.

31 R. Altkorn, I. Koev and M. J. Pelletier, Appl. Spectrosc., 1999, 53, 1169-1176.

32 R. Altkorn, I. Koev, R. P. Van Duyne and M. Litorja, Appl. Opt., 1997, 36, 8992-8998.

33 M. Brzozowski, M. O’Brien, S. V. Ley and A. Polyzos, Acc. Chem. Res., 2015, 48, 349-362.
34 S. Ponce, H. Christians, A. Drochner and B. J. M. Etzold, Chem. Ing. Tech., 2018, 90, 1855-1863.

35 C. Sambiagio and T. Noël, Trends Chem., 2020, 2, 92-106.

36 WPI, 4000 Series Liquid Waveguide Capillary Cell, Instruction Manual, 2014.

37 G. Dlubek, J. Pionteck, K. Rätzke, J. Kruse and F. Faupel, Macromolecules, 2008, 41, 6125-6133.

38 A. Roibu, S. Fransen, M. E. Leblebici, G. Meir, T. Van Gerven and S. Kuhn, Sci. Rep., 2018, 8, 1-10.

39 M. Irie, K. Sakemura, M. Okinaka and K. Uchida, J. Org. Chem., 1995, 60, 8305-8309.

40 T. Sumi, Y. Takagi, A. Yagi, M. Morimoto and M. Irie, Chem. Commun., 2014, 50, 3928-3930.

41 M. O'Brien, I. Baxendale and S. Ley, Synfacts, 2010, 2010, 1199-1199.

42 A. Alvarez-Martin, S. Trashin, M. Cuykx, A. Covaci, K. De Wael and K. Janssens, Dyes Pigm., 2017, 145, 376-384.

43 B. W. J. Pirok, G. Moro, N. Meekel, S. V. J. Berbers, P. J. Schoenmakers and M. R. van Bommel, J. Cult. Herit., 2019, 38, 29-36.

44 N. Kuramoto and T. Kitao, Dyes Pigm., 1982, 3, 49-58.

45 C. Weyermann, D. Kirsch, C. Costa-Vera and B. Spengler, J. Forensic Sci., 2009, 54, 339-345.

46 G. Favaro, D. Confortin, P. Pastore and M. Brustolon, J. Mass Spectrom., 2012, 47, 1660-1670. 